\title{
Long-Range Dichoptic Interactions in the Human Visual Cortex in the Region Corresponding to the Blind Spot
}

\author{
SRIMANT P. TRIPATHY,* DENNIS M. LEVI*†
}

Received 16 July 1993; in revised form 8 October 1993

\begin{abstract}
The region of the visual field of one eye that corresponds to the blind spot of the contralateral eye is believed to be monocular. We measured dichoptic contour interaction in this region of the visual field in humans by having observers report the orientation of a test letter " $T$ " presented to this region, in the presence of fianking T's presented around the blind spot of the fellow eye. A large drop in performance was seen because of the flanks, showing clearly the existence of dichoptic contour interaction in this "monocular" region of the visual field. This suggests that the cortical representation of the region of the visual field that corresponds to the contralateral eye's blind spot is not strictly monocular. The absence of direct retinal afferents from one eye to this region of cortex suggests the involvement of horizontal cortical connections in the contour interaction phenomenon. Our estimates of the extent of contour interaction in mm of striate cortex are comparable to the reported lengths of the long-range horizontal connections in the striate cortex of monkeys. Our results are consistent with the proposition that long-range horizontal connections of the striate cortex may mediate the contour interaction phenomenon.
\end{abstract}

Blind spot Contour interaction Long-range horizontal connections Scotoma

The visual scene is visuotopically mapped with great precision onto the lateral geniculate nucleus (LGN) and striate cortex (Pearlman, 1987). For the most part the visual field is represented binocularly and most cortical cells receive excitatory inputs from the two eyes (Poggio \& Fischer, 1977). However, there are two regions of the visual field that are notable exceptions with regard to binocularity: the optic disk or blind spot and the monocular crescent of each eye. The physiological blind spot in normal observers can, for most purposes, be considered to be a naturally occurring monocular retinal scotoma. A great deal of recent interest has been generated by studies in humans involving scotomas either physiological (Ramachandran, 1992), artificial (Ramachandran \& Gregory, 1991; Ramachandran, Gregory \& Aiken, 1993) or pathological (Fendrich, Wessinger \& Gazzaniga, 1992). In addition, several studies have induced retinal lesions in animals in order to examine any resulting cortical reorganization (Chino, Kaas, Smith, Langston \& Cheng, 1992; Gilbert, 1992). Studying the naturally occurring scotoma can provide valuable insight into understanding the neural organization that occurs at such retinal discontinuities and may help

*College of Optometry, University of Houston, Houston, TX 77204 6052, U.S.A.

†To whom all correspondence should be addressed. to predict the performance of human observers with pathological scotomas of retinal or cortical origin.

In humans the optic disk is an elliptical region on the retina that is reported to correspond, in terms of visual angle, to a vertical height of 7-8 deg and a width of 5-6 deg (Le Grand, 1967; but see Table 1). It is located on the nasal retina of each eye, with its inner edge approximately $13 \mathrm{deg}$ from the fovea radially and approximately 2-3 deg below the foveal center. It is a region with no photoreceptors and is a naturally occurring retinal lesion or scotoma (Le Grand, 1967). Since the blind spots are on the nasal hemi-retinae of the two eyes, the visual hemi-field containing each blind spot is topographically mapped onto the LGN and the visual cortex in the contralateral hemisphere of the brain (Pearlman, 1987).

Each LGN in primates is known to consist of six layers. Using the conventional system of numbering the layers, 1, 4 and 6 are the layers driven by the contralateral eye, while layers 2,3 and 5 are driven by the ipsilateral eye. In mammals, the different layers of the LGN are in precise topological register with one another (Bishop, Kozak, Levick \& Vakkur, 1962; Pearlman, 1987). In primates the contralateral layers are discontinuous at the locations that correspond to the blind spot. These discontinuities in the layers are seen as cell free regions. Hence, the projection of the blind spot onto 
the LGN passes through the LGN without intersecting any of the contralateral layers (Malpelli \& Baker, 1975). Similar discontinuities in the contralateral layers of the LGN have been reported in a variety of mammalian species (Kaas, Guillery \& Allman, 1973). Thus, in the contralateral layers of the LGN of mammals, the representation of the blind spot seems to be non-existent (or it exists as a gap).

In each hemisphere of the brain, the region of the striate cortex that represents the contralatcral eye's blind spot is believed to be strictly monocular. This belief is based on 2-deoxyglucose experiments conducted on monkeys (Kennedy, Des Rosiers, Jehle, Reivich, Sharpe \& Sokoloff, 1975; Kennedy, des Rosiers, Sakurada, Shinohara, Reivich, Jehle \& Sokoloff, 1976; Horton, 1984; LeVay, Connolly, Houde \& Van Essen, 1985). The blind spot is represented as a disk in the striate cortex, driven entirely by the ipsilateral eye and devoid of ocular dominance columns. Ocular dominance columns are seen outside of this disk, with adjacent columns being dominated by the opposite eyes (LeVay et al., 1985). Horton (1984) also looked to see if some neural filling-in process might exist in this region on account of activity outside of layer IV. He concluded ". . . to the extent that the 2-deoxyglucose technique accurately refiects local neuronal activity, the optic disc representation in all layers is purely monocular, metabolically and physiologically."

While the techniques used in the studies cited above would uncover macroscopic structural components in the brain, they may not have the resolution necessary to uncover small localized details. For example, horizontal connections extending as far as $6-8 \mathrm{~mm}$ have been reported in cat and monkey striate cortex (Gilbert \& Wiesel, 1979, 1983; Martin \& Witteridge, 1984; Callaway \& Katz, 1990; Gilbert, 1992). It is possible that horizontal connections could exist in the region of striate cortex representing the blind spot without being detected by the 2-deoxyglucose experiments. If these long-range connections exist in the cortical region representing the blind spot and if they are binocular in nature, then this region of striate cortex cannot be strictly monocular as is currently believed. The goal of the present study is to test psychophysically whether binocular interactions exist in the region of the cortex that corresponds to the blind spot in humans by measuring dichoptic contour interaction using stimuli that would be expected to stimulate this "monocular" cortical region.

Visual performance is degraded when additional contours are placed in the neighborhood of the stimulus used to measure performance. This degradation of performance in the presence of flanking contours is termed contour interaction (Flom, Weymouth \& Kahneman, 1963; Bouma, 1970). Contour interaction extends radially from the test stimulus as far as half the eccentricity of the test stimulus (Bouma, 1970; Andriessen \& Bouma, 1976; Toet \& Levi, 1992). It has been reported in a variety of visual tasks, e.g. letter discrimination (Bouma, 1970; Toet \& Levi, 1992; Nazir, 1992), tilt discrimination (Andriessen \& Bouma, 1976; Westheimer, Shimamura \&
McKee, 1976) and vernier acuity (Westheimer \& Hauske, 1975; Levi, Klein \& Aitsebaomo, 1985). It is believed to be a cortical phenomenon because performance is seen to be similar when flanking contours are placed in either the same eye as the test stimulus or in the opposite eye (Flom, Heath \& Takahashi, 1963; Westheimer \& Hauske, 1975). In addition, the degree of interaction between contours is affected by how similar in size and shape the flanking contours are to the contours of the test stimulus (Nazir, 1992; Kooi, Toet, Tripathy \& Levi, 1994). Since LGN neurons are not very specific with regard to shape and orientation (they have roughly circular -hence only minimally oriented-receptive fields), the specificity of contour interaction to the nature of the flanks, along with the dichoptic nature of contour interaction, suggests that the origins of contour interaction are cortical. The cortical long-range horizontal connections discussed earlier have been suggested to provide the neural substrate for lateral contour interactions (Gilbert \& Wiesel, 1990; Gilbert, Hirsch \& Wiesel, 1990; Hirsch \& Gilbert, 1991; Gilbert, 1992). Thus, contour interaction may be a useful phenomenon for studying binocular interaction in the cortical representation of the blind spot.

The question we address is: can contours presented around the blind spot of one eye interact dichoptically with contours presented to the other eye, in the region corresponding to the first eye's blind spot? To anticipate, we find strong dichoptic contour interaction between contours presented to one eye in the region corresponding to the second eye's blind spot and contours presented around the second eye's blind spot. This result suggests that the cortical representation of the blind spot cannot be strictly monocular as it is currently believed to be. Our results are consistent with the proposition that long-range horizontal connections in the striate cortex may be involved in the contour interaction process.

\section{METHODS}

Observers were asked to report the orientation of a test letter " $T$ " in the presence of flanking Ts. In one set of experiments, the test $T$ was presented to the left eye in the lower visual field at an eccentricity equal to that of the center of the blind spot. In a second set of experiments the test $T$ was centered at the point in the visual field of the left eye that corresponded to the center of the blind spot of the right eye. In each case, flanking Ts were presented either to the same eye or to the opposite eye, at varying separations from either the center of the test $T$ or the location in the right eye that corresponded to the center of the test $T$. For each experimental condition, the percentage of correct responses was determined as a function of test to flank separation. These scores were used to estimate the extent of contour interaction. The two authors and one naive observer participated in the experiment. None of the observers had any known visual abnormalities. 


\section{Stimuli}

The stimuli were generated on an IBM PC clone fitted with an Orchid ProDesigner II SVGA board and were presented on a Sony multiscan monitor. To enable viewing of dichoptic images a front surface mirror $(20 \mathrm{~cm}$ by $20 \mathrm{~cm})$ was mounted vertically, perpendicular to the mid-line of the monitor, in the observer's septal plane, with the mirrored surface facing to the right of the observer [see schematic in Fig. 1(E)]. All experiments were performed under binocular viewing conditions and used the mirror. A black occluder was suspended before the right half of the computer monitor to occlude the direct image of the monitor. The occluder was in a plane almost parallel to the plane of the monitor screen and separated from the monitor screen by approximately $15 \mathrm{~cm}$. A small gap was provided between the mirror and the occluder. This gap between the occluder and the mirror was adjusted for each observer so that only the laterally inverted reflection of the right half of the computer screen was visible to the observer's right eye, through the gap. The left eye viewed the left half of the screen directly. Chin and forehead rests were used in all experiments in order to minimize head movements. Nonius lines and binocular fixation points were provided.

In the main experiment the test stimulus consisted of a letter $\mathrm{T}$ presented to the left eye at a location in the visual field that corresponded to the center of the previously mapped blind spot of the right eye. The mapping of the blind spot onto the monitor screen is discussed later in this section. The orientation of the test $T$ was randomly selected from one of the cardinal orientations: up, down, left or right. Along with the test $\mathrm{T}$ presented to the left eye, flanking Ts were presented simultaneously to the right eye, on most of the trials [Fig. 1(A)-henceforth referred to as the blind spot dichoptic condition]. The flanks consisted of three Ts equidistant from the center of the blind spot of the right eye. The three Ts were located one above, one below and one to the left of the center of the blind spot. A flanking $T$ in the fourth direction was not used because of limitations imposed by the size of the monitor's screen. Trials in which the test Ts were presented without any flanks were randomly interleaved with flanked trials. On each flanked trial, the distance of the flanks from the center of the blind spot was selected randomly from five previously determined distances. On these trials, the orientation of each flanking $\mathrm{T}$ was randomly selected from the four cardinal orientations.

The experiment was also performed with the test and all the flanking Ts presented to the left eye [Fig. 1(B)henceforth the blind spot monocular condition]. The test $T$ was presented to the same location as the earlier condition. The flanks in a trial were all equidistant from the test T. As before, the test-to-flank separation was varied from trial to trial and unflanked trials were randomly interleaved. The septal plane mirror, binocular fixation points and binocular nonius lines were used as before.

In another set of control experiments performed in the lower visual field, both test and flanks were presented to opposite eyes in one condition and to the same eye in a second condition [Fig. 1(C) and (D)-henceforth the lower (or inferior) visual field dichoptic and monocular conditions respectively]. The eccentricity at which the test $T$ was presented was the same as the eccentricity of the center of the blind spot determined individually for each observer. The "missing" fourth flank was the one closer to the fovea. In other respects, the control experiments were identical to the main experiment.

In all the experimental conditions described so far, the flanks were either centered around the test or around the location in the other eye that corresponded to the test. Another simple control experiment was performed to see the effect of the three "flanks" when the test and "flanks" are centered around non-corresponding locations. Here the word "flanks" is clearly inappropriate since the "flanks" are not really spatially adjacent to the test. Hence, when describing this condition the word is enclosed in quotes. The stimuli were identical to those used in the lower visual field conditions, with the difference being that the test was displaced $6 \mathrm{deg}$ to the left and the center of the "flanks" was displaced $6 \mathrm{deg}$ to the right, the displacement of the flanks being performed monocularly in one condition and dichoptically in a second condition. These two conditions are henceforth referred to as the non-corresponding loci monocular and dichoptic conditions respectively.

The experiments were performed in a dimly lit room. Viewing distance was approximately $26 \mathrm{~cm}$. A background grid of horizontal and vertical lines $1.7 \mathrm{deg}$ apart and extending $33 \mathrm{deg}(\mathrm{H})$ by $23 \mathrm{deg}(\mathrm{W})$ was provided in order to assist accommodation and fusion. The test and flanks were displayed in a $20 \mathrm{deg}(\mathrm{H})$ by $16.7 \mathrm{deg}(\mathrm{W})$

FIGURE 1 (overleaf). Schematics of the stimulus configurations used in the experiments and the experimental setup used to display the stimuli dichoptically. Contained within each large circle in (A)-(D) is the stimulus presented to each eye under the different experimental conditions used. The dark ellipses represent the observer's blind spot and were not a part of the stimulus. The shaded ellipses also were not part of the stimulus and represent the region of the left eye's visual field that corresponds to the right eye's blind spot. Dichoptic foveal fxation points and nonius lines were provided as seen in the left half of each large circle. The binocular percept is shown in the rectangle on the right. The blind spot dichoptic condition is illustrated in (A). Here the binocular percept corresponds to a test-to-flank separation larger than the radius of the blind spot. (B), (C) and (D) illustrate the biind spot monocular, the lower visual field dichoptic and the lower visual field monocular conditions respectively (see text for details). (E) A schematic of the setup used to display dichoptic images, viewed form above. The septal plane mirror superimposed (perceptually) the laterally inverted image of the right half of the screen over the left half of the screen. The occluder prevented direct viewing of the right half of the screen. A ray of light is traced from corresponding points in the two halves of the screen. 
A DICHOPTIC - BUND SPOT
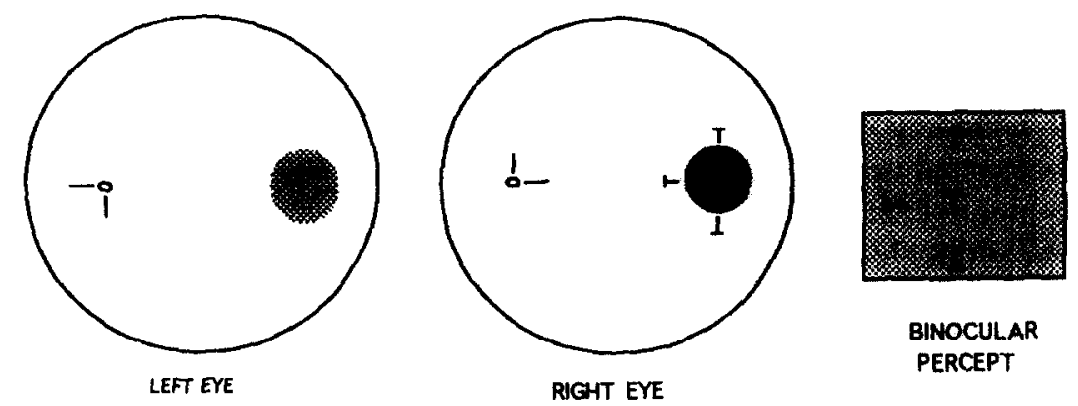

B MONOCULAR - BUND SPOT
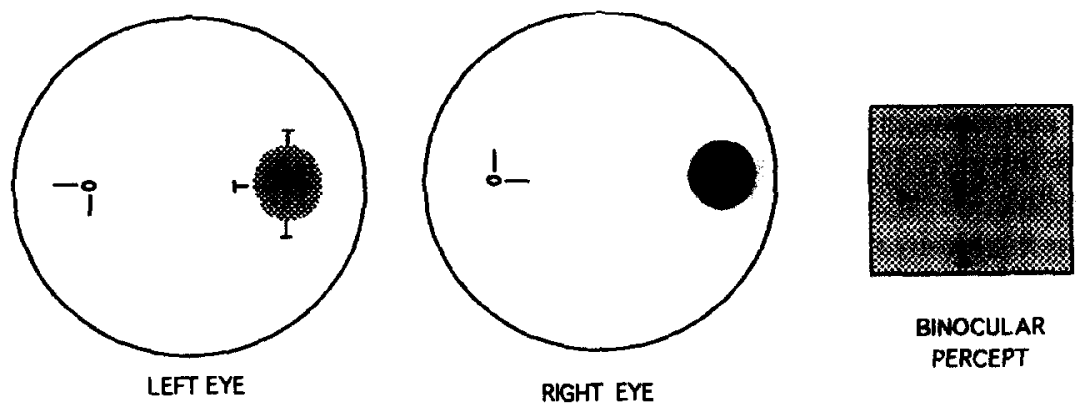

C DICHOPTIC - LOWER VSUAL FELD

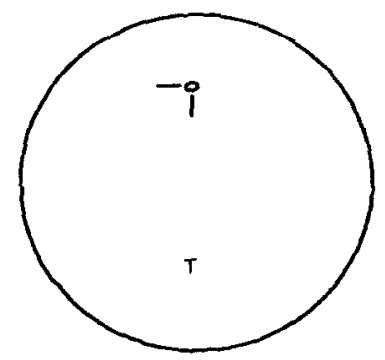

LEFT EYE

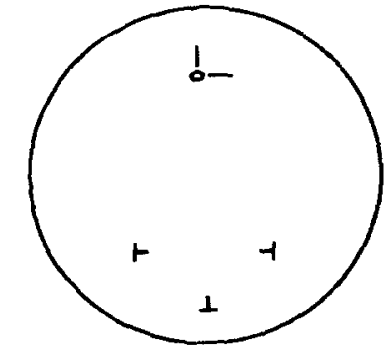

RIGHT EYE

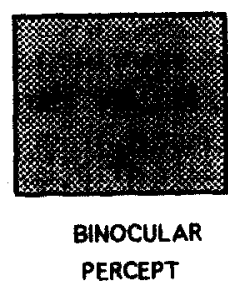

PERCEPT

D MONOCULAR - LOWER USUAL FIELD
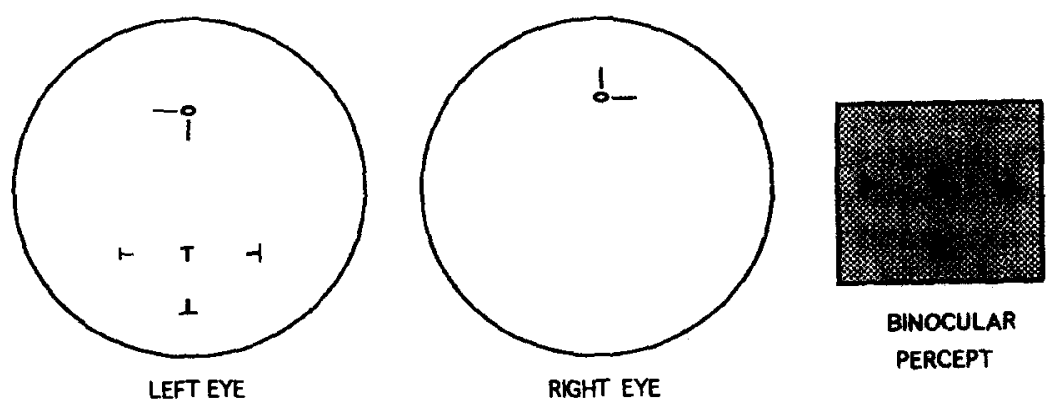

BINOCULAR PERCEPT

E SCHEMATIC OF SETUP

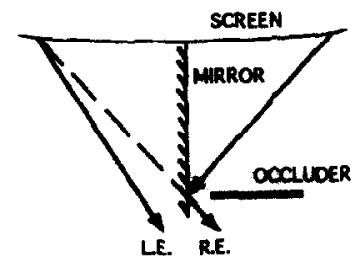

FIGURE 1. Caption on previous page. 
grid-free region in the right-middle of the gridded portion of the screen. Dichoptic fixation points and foveal nonius lines were provided in order to ensure proper binocular alignment of the eyes. The test and flanking Ts were white (intensity $=112 \mathrm{~cd} / \mathrm{m}^{2}$ as measured by a Pritchard Spectra photometer, contrast $=76 \%$ ) on a gray background (intensity $=15 \mathrm{~cd} / \mathrm{m}^{2}$ ). The size of the Ts was adjusted so that all observers could correctly identify the orientation of the isolated (unflanked) $\mathrm{T}$ more than $90 \%$ of the time. The size of each stroke of the resulting $T$ was 50 arcmin by $10 \mathrm{arcmin}$. All stimuli were displayed for $300 \mathrm{~ms}$. Between trials, the test-to-flank separation (center-to-center distance) was varied over the range $1.6-9.0 \mathrm{deg}$.

\section{Procedure}

During the experiments, the observer sat with his/her chin on the chin rest, fixated on the fixation point provided to each eye and attempted to keep the nonius lines aligned at all times. The presentation of each stimulus was initiated by the observer. When the fixation points in the two eyes were binocularly fused and the nonius lines were aligned, the observer initiated a trial by pressing a key. The test and flanking Ts were immediately displayed on the screen for a duration of $300 \mathrm{msec}$. Within a run the flanking Ts were either all presented to the eye to which the test $\mathrm{T}$ was presented (monocular), or were all presented to the opposite eye (dichoptic). The observer reported the orientation of the test $T$ by pressing one of four keys, where each key was designated to one of the four orientations. The computer provided feedback following each trial and tallied the responses at the end of each run.

Each run of the experiment tested one of the six conditions: the blind spot dichoptic condition, the blind spot monocular condition, the lower field dichoptic condition, the lower field monocular condition, the noncorresponding loci dichoptic condition or the non-corresponding loci monocular condition. A run consisted of 10 practice trials and 140 recorded trials. The 140 recorded trials consisted of 100 flanked trials and 40 unflanked trials. The 100 flanked trials were distributed equally over the five test-flank separations. Five runs were performed for each condition, yielding 100 repetitions for each test-flank separation and 200 repetitions for the unflanked trials.

Prior to the experiments, the center of the blind spot of the right eye of each observer was mapped onto the computer screen. From this, the position of the point in the left eye that corresponded to the center of the blind spot of the right eye was determined. This mapping was also used to determine the point where the test $T$ and flanks would be presented in the lower visual field. The next section details the procedure used to determine the center of the observers blind spot and the corresponding point in the fellow eye.

\section{Mapping the blind spot}

The setup for mapping the blind spot was identical to the arrangement used in the experiments. A red circle of diameter $3.5 \mathrm{deg}$ was displayed on the right half of the computer monitor. The observer could move this red circle either up, down, left or right, in steps of 25 arcmin by pressing appropriate keys on the keyboard. The observer viewed the reflection of the red circle in the septal plane mirror with his/her right eye, while keeping the dichoptic fixation square fused and the nonius lines aligned. While maintaining proper eye position, the observer adjusted the position of the red circle until it disappeared completely inside his/her right eye's blind spot. Once the circle had completely disappeared, the vertical height was kept fixed. The observer moved the red circle horizontally in one direction till he/she just saw the edge of the red circle. At that point the position of the red circle was recorded. The observer then reversed the direction of the circle and moved it until it was seen at the opposite margin of the blind spot and this position was again recorded. Three readings were taken at each margin of the blind spot and the mean of these readings was taken to be the horizontal mid-point of the blind spot. Similarly, the vertical mid-point of the blind spot was located by having the observer move the circle upwards and downwards along a vertical line, keeping its horizontal position fixed. The horizontal and vertical mid-points of the blind spot in the right eye were measured with respect to the position of the right eye's fixation spot. The position of the center of the blind spot in the right eye of each of the three observers is listed in Table 1. The point in the left eye's visual field that was the same horizontal and vertical distances from fixation was taken to correspond to the center of the blind spot of the right eye. For all observers, when the test was presented to the left eye in the region measured to correspond to the right eye's blind spot and the flanks were presented to the right eye, in the region surrounding the blind spot, the test $T$ was seen roughly centered with respect to the flanks, verifying the correctness of the correspondence between the regions in the two eyes. For the lower visual field corresponding loci conditions the center of the test $T$ was located vertically below the fixation point at an eccentricity equal to that of the

TABLE 1. Locations and sizes of the observers' blind spots

\begin{tabular}{lcccc}
\hline Observer & $\begin{array}{c}x_{\text {mid }} \\
(\mathrm{deg})\end{array}$ & $\begin{array}{c}y_{\text {mid }} \\
(\mathrm{deg})\end{array}$ & $\begin{array}{c}\text { Width } \\
(\mathrm{deg})\end{array}$ & $\begin{array}{c}\text { Height } \\
(\mathrm{deg})\end{array}$ \\
\hline DL & 16.1 & -0.8 & 6.0 & 6.0 \\
HD & 16.9 & +0.8 & 6.0 & 6.5 \\
ST & 16.5 & -0.3 & 7.0 & 6.5 \\
\hline
\end{tabular}

Listed here are the locations of the center of the blind spot and the approximate size of the blind spot for all the observers tested. The center of the blind spot is specified in terms of the horizontal separation between the fovea and the center of the blind spot $\left(x_{\text {mid }}\right)$ and the vertical separation between the fovea and the center of the blind spot ( $\left.y_{\text {mid }}\right)$, the separation being measured in degrees of visual angle. Positive/negative values of $y_{\text {mid }}$ refer to points above/below the horizontal meridian. The width and height columns of the table refer to the maximum horizontal width and the maximum vertical height of the blind spot. 
center of the blind spot. The test was seen centered among the flanks in these conditions too.

The extent of the blind spot of the right eye of each observer was also mapped using a flashing $T$ at different locations in and around the blind spot. On each trial, the location of the test $\mathrm{T}$ was randomly selected from a grid of locations spaced one deg apart. For a complete mapping, each grid location was tested four times in random order. For each presentation of the flashing $T$ observers responded by pressing one key if the $T$ was visible and another key if it was not visible. A point was assumed to he inside the blind spot, if a $T$ centered at that point was visible on fewer than three of the four presentations. The blind spot was plotted on linear graph paper and its maximum width and the maximum vertical height were measured. Table 1 lists these maximum widths and heights. The centroid of the graphed blind spot was also measured and this corresponded very closely (within $0.5 \mathrm{deg}$ horizontally and vertically) to the center of the blind spot determined by the earlier method of moving the red dot.

\section{RESULTS}

\section{(A) Contour interaction as a function of flank separation}

We first report the results for the contour interactions observed when the test and flanks are presented over intact retina in the lower visual field, followed by the results of the main experiment, where the flanks were presented in and around the blind spot of one eye and the test in the corresponding region in the other eye.

(i) Contour interaction in the lower visual field conditions. Figure 2 shows, for two observers, the variation of performance scores (the percentage of correct identifcations of the test $T$ ) with test-to-flank separation, when the test and flanks were presented in the lower visual field. The error bars represent \pm 1 standard error of the mean (SEM) as determined using the Binomial Theorem. When no flanks were presented (rightmost symbol) performance scores were very close to $100 \%$. When flanks were presented, either in the monocular or dichoptic condition, performance scores dropped dramatically, reaching close to chance $(25 \%)$ at the smallest separations. This drop in performance in the presence of flanking contours is the contour interaction effect in the normal periphery. Foveal contour interaction has been reported to be similar under monocular or dichoptic conditions (Flom et al., 1963; Westheimer \& Hauske, 1975) and Fig. 2 extends this result to the peripheral visual field (see also Kooi et al., 1994). Monocular and dichoptic interactions were found to differ slightly at small separations for observer ST, with less interaction being observed for the dichoptic case. The reason for this discrepancy was not clear. Observer HD showed strong interaction over a larger range of separations. Both observers reported being unable to discriminate to which eye the flanks were presented.

(ii) Contour interaction in the blind spot conditions. Figure 3 shows, for three observers, the performance scores as a function of test-to-flank separation, for the
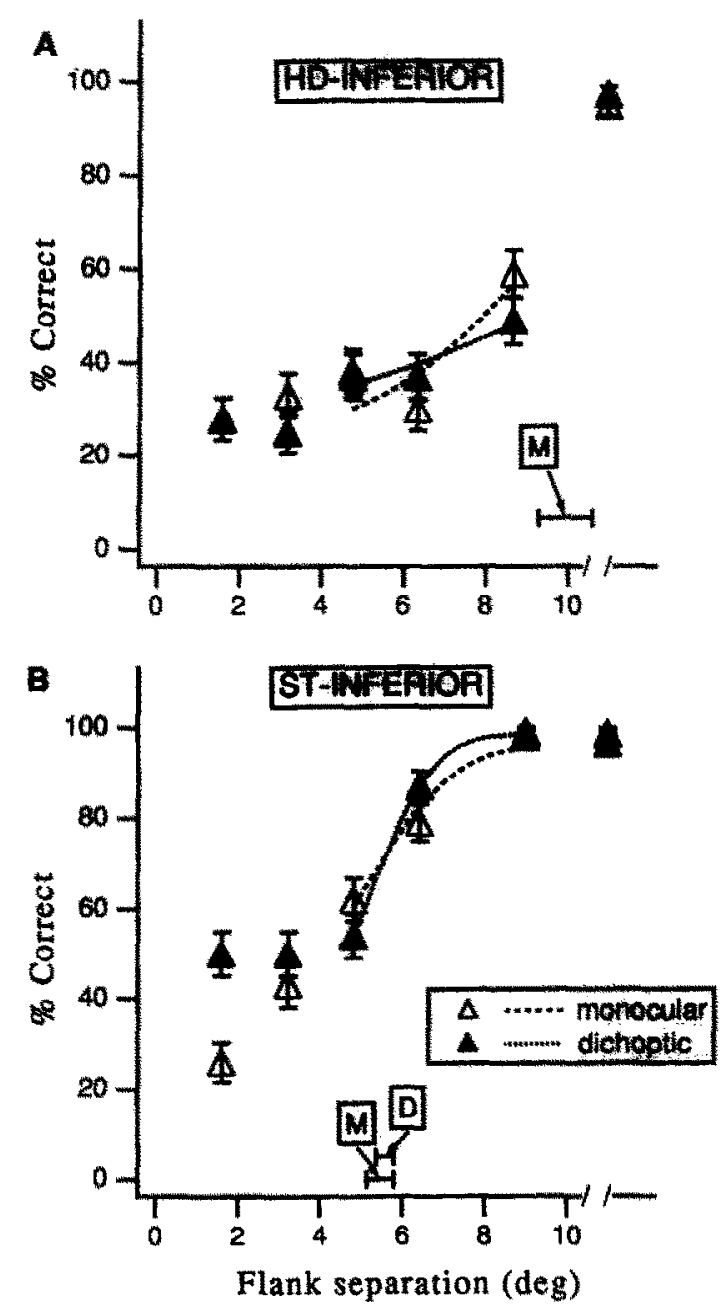

FIGURE 2. Performance scores as a function of test-to-flank separation for the lower visual field conditions for observer $H D(A)$ and observer ST (B). Solid symbols represent the data for the dichoptic condition while open symbols represent the data for the monocular condition. The flank separation is the distance between the center of the test $T$ and the center of each fanking $T$. The symbols at the largest flanking separation represent the performance for the unflanked trials. $N=100$ for each data point. Error bars represent \pm 1 SEM. The dotted lines are cumulative normal Gaussian fits (see section B of Results) to the data. Estimated extents of contour interaction (arrows-see section $B$ of Results) along with their associated SEMs are shown for the monocular (M) and dichoptic (D) conditions. HD's dichoptic extent of contour interaction was $13.7 \pm 3.1 \mathrm{deg}$ and is beyond the range of flank separations illustrated in this figure. Dichoptic and monocular performances are very similar except at small separations for observer ST.

cases when the test stimuli were presented to the left eye at the point in the visual field corresponding to the center of the right eye's blind spot. Error bars show \pm 1 SEM, as determined using the Binomial Theorem. The monocular performance is similar to that observed in the lower visual field, with performance being degraded almost to chance level at the smallest separations for all observers. However, the most interesting result is that strong contour interactions are observed when the flanks are dichoptically presented around the blind spot. The performance scores dropped from the unflanked per. formance score of nearly $100 \%$ to a minimum of about 65,55 and $35 \%$ for observers $\mathrm{DL}, \mathrm{HD}$ and $\mathrm{ST}$ respectively, for this dichoptic blind spot condition. This drop 
in performance very clearly shows that the region of the visual field of the left eye which corresponds to the right eye's blind spot strongly interacts dichoptically with the right eye. This surprising result suggests that the cortical
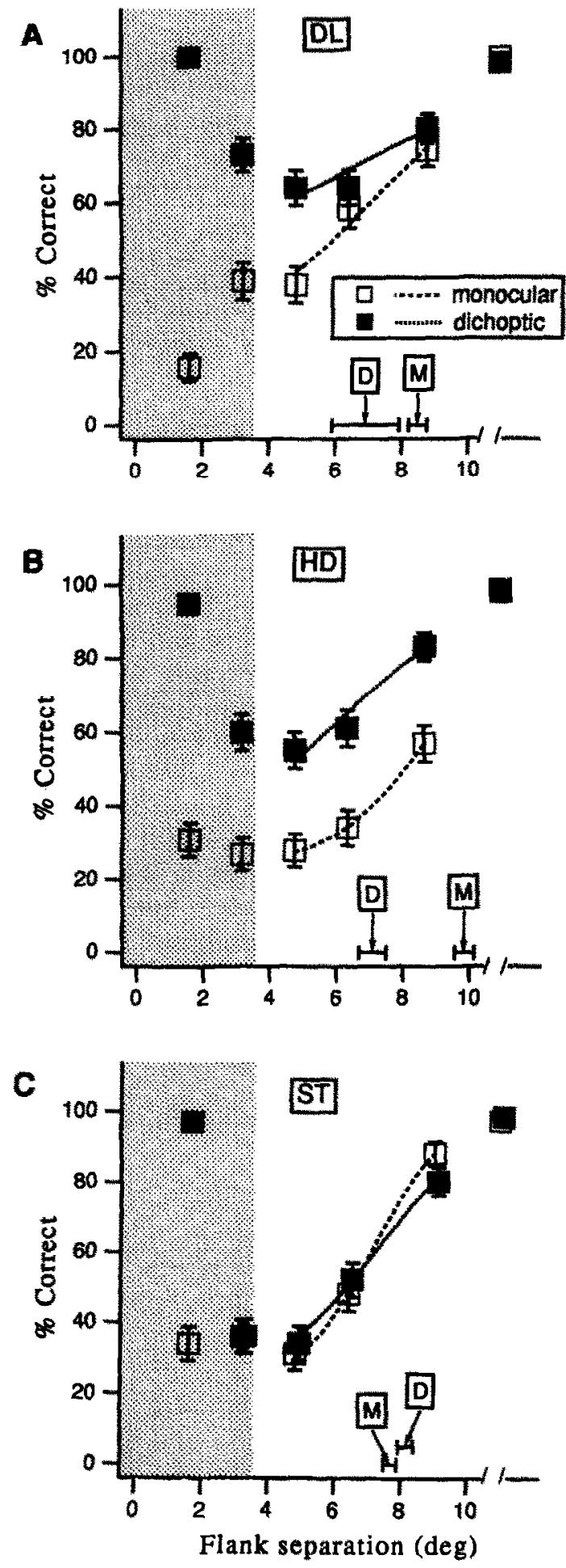

FIGURE 3. Performance scores as a function of test-to-flank separation for the blind spot conditions for observers HD (A), DL (B) and ST (C). See Fig. 2 legend for details. The stippled region indicates the approximate radius of the blind spot. Note the strong dichoptic contour interaction observed (the big drop in performance compared with the unflanked performance for the solid symbols) when flanks are presented around the blind spot (flank separations outside of the stippled region in figure). region representing the contralateral eye's blind spot must receive inputs from both eyes.

Dichoptic performance in the blind spot case was seen to be a U-shaped function of flank separation. At the smallest separation all of the flanks fell within the blind spot, and hence were not seen. At this separation, no contour interaction was observed and performance was seen to be comparable to the unflanked performance. At separations in the range 4-10 deg all the flanks were seen and strong contour interaction was observed. Monocular and dichoptic stimuli were indistinguishable, except when the flanks were presented at a separation that was approximately equal to the radius of the blind spot (the second smallest separation). At this separation, the dichoptically presented flanks appeared distorted, since they fell on the edge of the right eye's blind spot.

Eye movements were not monitored during these experiments. It could be argued that observers could have made substantial eye movements during the experiment such that the test $T$ was not really presented in a region that corresponds to the contralateral eye's blind spot, and so contour interaction was dichoptically observed. However, this could not have been the case, because if observers were making substantial anticipatory eye movements, these movements would also have occurred on some trials when the flanks were presented dichoptically at the smallest separation. If that were the case, observers would have seen the flanks on several of these trials. This would have resulted in a drop in performance at the smallest separation, on account of the resulting contour interaction. The fact that no drop in performance was observed at the smallest separation implies that the observers did not make substantial eye movements. Small eye movements would not have significantly affected our results, considering the size of the blind spot (almost 6 deg diameter), the eccentricity of the stimuli (almost $17 \mathrm{deg}$ ) and the large range of test-toflank separations over which the interactions were observed (about $8 \mathrm{deg}$ ). Furthermore, the stable results seen when plotting the size of the blind spot (the edges of the plotted blind spot were not seen to shift signifcantly between repetitions of plotting of the blind spotalso the flashed $T$ at any particular location was always seen or never seen except for a very small annular region where it was only sometimes seen) showed that all the observers were clearly capable of maintaining fixation well within the limits required in this experiment.

(iii) Contour interaction for the non-corresponding loci conditions. In both the monocular and the dichoptic conditions very little contour interaction was seen when test and "flanks" were centered around non-corresponding loci (Fig. 4). A slight decrease in performance was seen as the separation between the "flanks" was increased. This was because as the separation between the "flanks" was increased, one of the "flanking" Ts approached the test $T$. The decrease in performance can be attributed to the contour interaction between the test $T$ and the closest "flanking" $T$. At the largest separation of the "flanks", the test $T$ was about $5 \mathrm{deg}$ from the closest "flanking" $T$. This experiment demonstrates that 


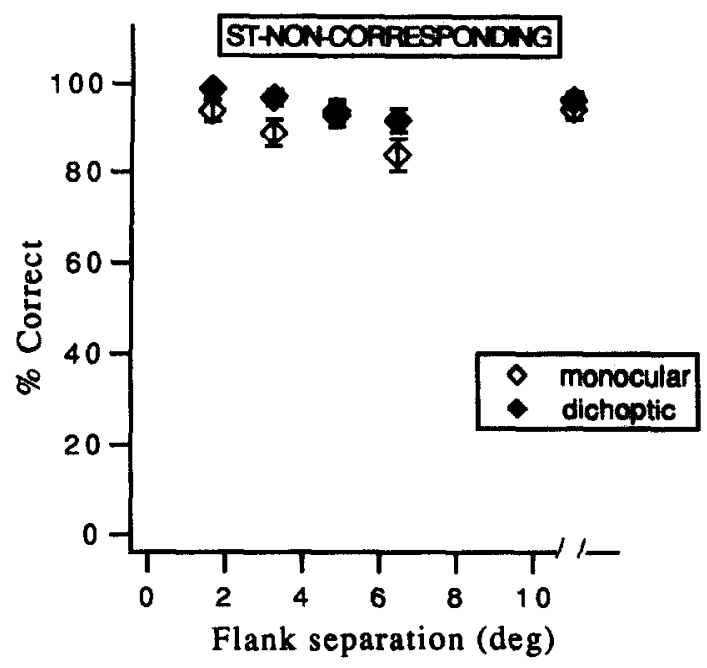

FIGURE 4. Performance scores as a function of test-to-flank separation for the non-corresponding conditions for observer ST. Little or no contour interaction is observed when distracting contour are presented at locations spatially separated from the test contours.

the strong interaction observed in the earlier experiments is a consequence of spatial proximity.

\section{(B) Estimating the spatial extents of contour interaction}

In order to determine the spatial extents of contour interaction, cumulative normal Gaussian functions were fitted to the data for each condition in which the test and flanks were centered around corresponding loci. The spatial extents of contour interaction for the different conditions were estimated from these fits.

Cumulative normal Gaussian functions were fitted to the data points having test-to-flank separations of approximately 5, 7 and $9 \mathrm{deg}$, for all the conditions tested, with the exception of the non-corresponding loci conditions. The upper and lower asymptotes of these fits were held at the unflanked performance score (close to $100 \%$ ) and chance performance $(25 \%)$ respectively. For the sake of consistency, in all the conditions where curve fitting was done, only the separations that were greater than the blind spot radius were used in the fits. All fits were performed using the Igor software package running on a Macintosh computer. Igor estimates the best fit to the data by minimizing the chi-square error between the actual data and the fits, using the Levenberg-Marquardt algorithm. The equation that was fit to the data was:

$$
\begin{aligned}
& y=100 *\left[0.25+a_{0}\left(1+\left(\operatorname { e r f } \left(\left(x-a_{1}\right) /\right.\right.\right.\right. \\
& \left.\left.\left.\left.\quad\left(\operatorname{sqrt}(2) * a_{2}\right)\right)-1\right) / 2\right)\right]
\end{aligned}
$$

where $x$ is the flank separation, $y$ is the performance score, $\operatorname{erf}(z)$ is the error function commonly used in signal detection theory and $a_{0}, a_{1}, a_{2}$ are parameters that correspond to the amplitude, the mean and the standard deviation of the cumulative normal distribution. The amplitude was held fixed at a value equal to the difference between the unflanked performance score and chance performance score. $a_{1}$ and $a_{2}$ were varied to get the best fit to the data. The best fits are shown as dotted lines in Figs 2 and 3. Table 2 lists the parameters found
TABLE 2. Parameters for cumulative normal Gaussian fits to the data

\begin{tabular}{ccccc}
\hline Condition & Observer & $a_{0}$ & $a_{1}$ & $a_{2}$ \\
\hline Lower field & HD & 0.70 & $9.02 \pm 0.58$ & $2.95 \pm 0.88$ \\
monocular & ST & 0.72 & $4.86 \pm 0.31$ & $1.85 \pm 0.55$ \\
Lower field & HD & 0.73 & $11.69 \pm 2.62$ & $6.40 \pm 3.50$ \\
dichoptic & ST & 0.74 & $5.17 \pm 0.19$ & $1.26 \pm 0.31$ \\
Blind spot & DL & 0.73 & $7.28 \pm 0.28$ & $3.27 \pm 0.59$ \\
monocular & HD & 0.74 & $9.07 \pm 0.28$ & $2.38 \pm 0.68$ \\
& ST & 0.72 & $7.16 \pm 0.21$ & $1.61 \pm 0.22$ \\
Blind spot & DL & 0.75 & $5.10 \pm 0.80$ & $6.06+1.68$ \\
dichoptic & HD & 0.73 & $5.91 \pm 0.38$ & $3.53 \pm 0.68$ \\
& ST & 0.73 & $7.35 \pm 0.23$ & $2.39 \pm 0.34$ \\
\hline
\end{tabular}

The parameters that provided the best cumulative normal Gaussian fit to the data for the different conditions and observers tested are listed here. $a_{0}, a_{1}$ and $a_{2}$ refer to the amplitude, mean and standard deviation of the cumulative normal Gaussian and their associated SEMs. There are no error estimates for $a_{0}$, since this parameter was held fixed.

for the best fit to the data for the different conditions tested.

The extent of contour interaction was taken to be the test-to-flank separation at which performance dropped from the unflanked performance by $1 / \mathrm{e}$ of the amplitude of the fitted cumulative Gaussian. The amplitude of the cumulative Gaussian for each condition was the difference between unflanked performance score for that condition (close to $100 \%$ ) and chance performance score $(25 \%)$. The arrows in Figs 2 and 3 represent the extent of the interaction. The horizontal error bars represent \pm 1 SEM as estimated from the fits to the data.

Figure 5 shows the extents of contour interaction in degrees of visual angle for the different observers for the different conditions tested. The error bars show 1 SEM as determined from the fitted Gaussians. The extents of interaction lie between 5 and $10 \mathrm{deg}$, for all conditions, with the exception of the inferior dichoptic condition for observer HD, for which the extent is $13.7 \mathrm{deg}$. The mean

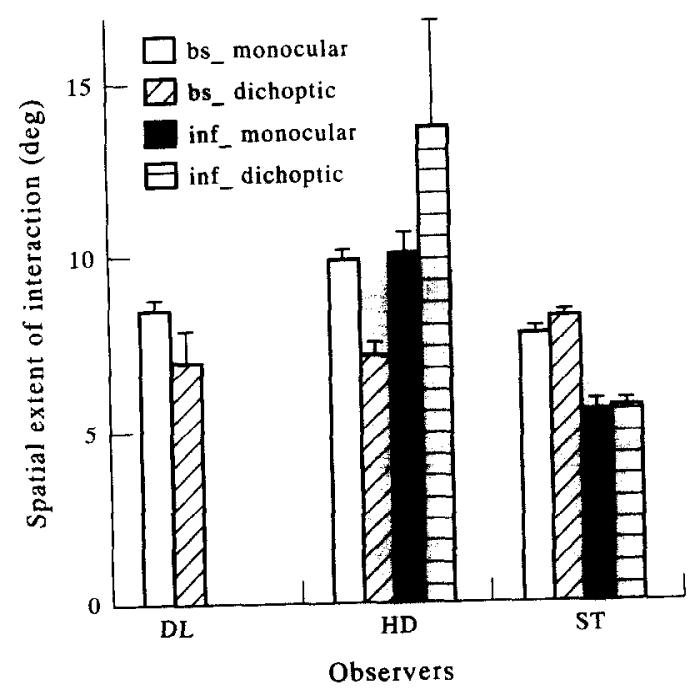

FIGURE 5. Estimates of the spatial extents of interaction for the different observers for the different conditions tested. Note that the spatial extents of interaction in the blind spot conditions are comparable to the extents of interaction in the lower visual field conditions (except for observer HD in the lower visual field dichoptic condition). 
extent of interaction is $8 \mathrm{deg}$, roughly 0.5 times the eccentricity $(16.5 \mathrm{deg})$ of the test stimulus. These extents of contour interaction are similar to those that have been reported in other studies of peripheral vision (Bouma, 1970; Andriessen \& Bouma, 1976; Toet \& Levi, 1992; Kooi et al., 1994). Clearly, the extent of dichoptic contour interaction in the region corresponding to the contralateral eye's blind spot is comparable to the extents of contour interaction seen at similar eccentricities in other rcgions of the visual field. This result provides strong evidence that the regions of the visual cortex that represent the two blind spots receive inputs from both eyes.

\section{DISCUSSION}

\section{(A) Estimating the cortical extent of contour interaction}

We estimated the cortical extents of contour interaction at the eccentricity of the blind spot from the observed spatial extents of contour interaction, for the different experimental conditions. Our estimates were based on several reported estimates of linear cortical magnification $(M)$ in the striate cortex of humans and monkeys. Here $M$ refers to the distance in $\mathrm{mm}$ of striate cortex that corresponds to $1 \mathrm{deg}$ of the visual field. $M$ decreases with eccentricity, with the dependency being almost linear. Our calculations of the extents of cortical interaction (described below) obtained from the more reliable estimates of $M$ were comparable to one another. In all these cases, the cortical extents were comparable to the sizes of the horizontal connections known to exist in monkey striate cortex.

The spatial extents of interaction at the eccentricity of the blind spot (about $16.5 \mathrm{deg}$ ) were seen to vary between 5 and $10 \mathrm{deg}$, with the exception of that for the lower visual field dichoptic condition for HD. The average extent of interaction was about $8 \mathrm{deg}$. These spatial extents of interaction were transformed to cortical distances using estimates of $M$ from monkeys and humans.

$M$ can be predicted from the equation (Levi et al., 1985; Van Essen, Newsome \& Maunsell, 1984; Drasdo, 1991):

$$
M=(1 / k) *\left(E+E_{2}\right)^{-1},
$$

where $E$ is the eccentricity of the test stimulus in deg (i.e. the eccentricity of the center of the blind spotabout $16.5 \mathrm{deg}$ ), $E_{2}$ is the eccentricity in deg at which cortical magnification has dropped to $0.5 * M_{\mathrm{f}}$ (the foveal magnification factor in $\mathrm{mm} / \mathrm{deg}$ ) and $k=\left(M_{\mathrm{f}} * E_{2}\right)^{-1}$. For the rhesus monkey the following parameters have been reported (Levi et al., 1985; Dow, Snyder, Vautin \& Bauer, 1981): $k=0.12 \mathrm{~mm}^{-1}$; $M_{\mathrm{f}}=10.4 \mathrm{~mm} / \mathrm{deg} ; E_{2}=0.8 \mathrm{deg}$. Using these values in (2), $M$ at 16.5 deg works out to be $0.48 \mathrm{~mm}$ of cortex/deg of visual space. This yields estimates of between 2.4 and $4.8 \mathrm{~mm}$ for the cortical extents of interaction with an average extent of about $4 \mathrm{~mm}$.

Another study suggested a similar expression for the variation of linear cortical magnification with eccentricity (LeVay et al., 1985) based on results reported in earlier studies (Hubel \& Wiesel, 1974; Hubel \& Freeman, 1977). According to this study the expression for $M$ for the macaque monkey is given by:

$$
M=10 *(E+0.82)^{-1} .
$$

Using this expression, $M$ at $16.5 \mathrm{deg}$ eccentricity was found to be $0.577 \mathrm{~mm} / \mathrm{deg}$. The corresponding cortical extents of interaction were estimated to vary between 2.9 and $5.8 \mathrm{~mm}$ of cortex, with a mean cortical extent of around $4.6 \mathrm{~mm}$.

LeVay et al. (1985) measured the area of the blind spot's representation in the striate cortex using two different methods of mapping, one computerized and one manual. They found the area of the blind spot to be 8.8 and $14 \mathrm{~mm}^{2}$ with the computerized method and manual method respectively. Assuming the cortical representation of the blind spot of a monkey to be circular, and ignoring cortical anisometries, the cortical diameter of the blind spot would be approximately 3.4 and $4.2 \mathrm{~mm}$ for the two methods of mapping the blind spot. (Actually the cortical representation of the blind spot is twice as long as it is broad, but we ignore this as a first approximation.) Assuming that the monkey blind spot corresponds to a circular region of visual space having a diameter of $5.5 \mathrm{deg}$, the above values of cortical diameter yield cortical magnification factors of 0.62 and $0.76 \mathrm{~mm} / \mathrm{deg}$. Based on these estimates, the average spatial extent of the interaction (approximately $8 \mathrm{deg}$ ) would translate to 5 and $6 \mathrm{~mm}$ of cortical distance. The range of the cortical extents of interaction would lie between 3 and $8 \mathrm{~mm}$. Thus all the three estimates of cortical magnification using parameters determined for monkeys yield cortical extents of interaction that are within the range of the reported size (up to $6-8 \mathrm{~mm}$ of cortex) of the horizontal connections in monkeys and cats (Gilbert \& Wiesel, 1983, 1989, 1992; Martin \& Witteridge, 1984; Callaway \& Katz, 1990; Gilbert, 1992).

The human striate cortex can be considered to be a scaled up version of the monkey striate cortex, with a linear scaling factor of 1.6 (Tolhurst \& Ling, 1988; Drasdo, 1991). Using this factor to scale the cortical extents of interaction derived for monkeys [using equation (2)], the estimates for cortical extents of interaction (for humans) at the eccentricity of the blind spot vary between 3.8 and $7.7 \mathrm{~mm}$ of cortex, with a mean of $6.4 \mathrm{~mm}$. Another proposed estimate for the ratio human cortical magnification factor/monkey cortical magnification factor is 1.44 (Levi et al., 1985; Drasdo, 1991). Using this estimate the extents of cortical interaction for humans would be smaller than the earlier estimates by about $10 \%$.

Estimates of cortical magnification for man and monkey vary widely between studies (for a recent review see Drasdo, 1991). However, the more reliable estimates of cortical magnification yield extents of contour interaction that are comparable to the sizes of the horizontal connections in the striate cortex. 
(B) Speculations on the neural substrates of contour interaction

In the LGN the inputs from the two eyes are segregated into the contralateral layers and the ipsilateral layers. Though most LGN neurons are monocular, several studies have reported binocular interaction between the ipsilateral layers and the contralateral layers of the LGN in cats (Singer, 1970; Sanderson, Bishop \& Darian-Smith, 1971; Schmielau \& Singer, 1977; Xue, Ramoa, Carney \& Freeman, 1987). A few studies have reported such interactions in monkeys (Marrocco \& McClurkin, 1979; Rodieck \& Dreher, 1979). However, the LGN is unlikely to be the location of interaction of contours in humans because:

(i) The binocular neurons in the primate LGN are few in number and appear to be localized to the magnocellular layers [in one study about $13 \%$ of the 91 neurons recorded from in monkey LGN were found to have binocular facilitation or inhibition (Marrocco \& McClurkin, 1979), another study found non-dominant suppression in six neurons out of $\mathbf{4 5}$ tested in monkeys LGN and all six were found in the magnocellular layers of LGN (Rodieck \& Dreher, 1979)]. Since contour interaction is more likely to involve the parvocellular pathway more than the magnocellular pathway (since the task in our experiment is related to visual acuity), the dichoptic contour interaction observed in our experiments probably do not involve the neurons that display non-dominant suppression in the magnocellular layers of the LGN.

(ii) The neurons in the LGN have relatively unoriented receptive fields [small bias in orientation tuning is known to exist in LGN neurons (Smith, Chino, Ridder, Kitagawa \& Langston, 1990)] and cannot account for the specificity of contour interaction to the relative sizes, spatial frequencies, orientations and depths of test and flanking stimuli (Andriessen \& Bouma, 1976; Nazir, 1992; Polat \& Sagi, 1993; Kooi et al., 1994).

(iii) The binocular neurons in cat LGN become less responsive when the striate cortex is reversibly cooled, suggesting a cortical involvement in the observed binocularity in the LGN (Schmielau \& Singer, 1977).

Furthermore, it is not evident that binocular interactions in the LGN could explain our blind spot dichoptic interaction data because we are not aware of any studies that have reported binocular neurons within the LGN's representation of the region of the visual field that corresponds to the contralateral blind spot.

The striate cortex seems to be the earliest location within the visual pathway where connections suitable for contour interaction have been found. The long-range horizontal connections that have been reported in cat and monkey striatc cortex appear to be a reasonable substrate for contour interaction because:

(i) the extent of contour interaction expressed in $\mathrm{mm}$ of cortex appears to be similar in dimension to the extent of these horizontal connections (up to $6-8 \mathrm{~mm}$, Gilbert \& Wiesel, 1979, 1983; Gilbert, 1992),

(ii) horizontal connections are reported to connect together several cells with similar orientation response characteristics (Gilbert \& Wiesel, 1989). In addition, Wiesel and Gilbert (1988) and Van Essen, De Yoe, Olavarrin, Knierim, Fox, Sagi and Julesz (1988) have shown that the responses of orientation selective cells in striate cortex can be suppressed by similarly oriented elements well outside the "classical" receptive fields of these cells. Contour interactions too are stronger when the flanking contours have spatial characteristics similar to the spatial characteristics of the test contours (Nazir, 1992; Kooi et al., 1994).

Earlier studies have reported that the representation of the blind spot in the striate cortex is strictly monocular (Kennedy et al., 1975, 1976; Horton, 1984; LeVay et al., 1985). However, these studies are based on 2-deoxyglucose experiments which may not have the required resolution to uncover the long-range horizontal connection, if they exist in the cortical representation of the blind spot. Hence these studies cannot rule out binocular interactions within the striate cortex's representation of the blind spot.

Other possibilities are that contour interaction could occur at a site in the visual pathway that is beyond the striate cortex, or part of the interaction could occur in the striate cortex and part of it beyond the striate cortex. Our experiments cannot distinguish between these possibilities. However, they clearly demonstrate binocular interaction in the region corresponding to the blind spot, and this interaction is probably cortical. This provides a ncurophysiological prediction that if the striate cortex is the locus of contour interaction, then long-range horizontal connections must exist within the cortical representation of the blind spot. If long-range horizontal connections cannot be found within the striate cortex's representation of the blind spot, then the locus of contour interaction must lie beyond the striate cortex. Existence of the long-range connections within the striate cortex's representation of the blind spot would not however prove the striate cortex's involvement in the contour interaction process. While existence of these connections would be consistent with the striate cortex's involvement, the absence of such connections would provide strong evidence against the involvement of the striate cortex. (See note added in proof).

\section{(C) Is the physiological blind spot "sewn-up"",}

A straight line that grows longer to pass through the blind spot is perceived to be shorter than a similar straight line that grows longer but passes outside the blind spot (Andrews \& Campbell, 1991). A straight line that passes through the blind spot is seen to be shorter than a similar straight line in the other hemi-retina of the same eye at the same eccentricity or a similar straight line at the corresponding location in the other eye (Tripathy \& Levi, 1993; Tripathy, Levi \& Ogmen, 1994; see also Sears \& Mikaelian, 1994). However, these length distortions are evident only if the straight line extends less than about 2 deg on either side of the blind spot (Ferree \& Rand, 1912; Tripathy, Levi \& Ogmen, 1994). These experiments suggest that points that are on diametrically 
opposite sides of the blind spot may be represented adjacently in the cortex, i.e. sewn-up (beyond the striate cortex).

Other experiments suggest that points that are on diametrically opposite sides of the blind spot may not be cortically adjacent to each other. Two-dot alignment thresholds across the blind spot are never lower than alignment thresholds at comparable locations in the visual field, when the separation of the dots is kept constant (Tripathy, Levi \& Ogmen, unpublished results). Since two-dot alignment thresholds are known to decrease with reduction of the separation of the dots (Sullivan, Oatley \& Sutherland, 1972; Levi, Klein \& Aitsebaomo, 1985; Beck \& Halloran, 1985), this result suggests that either the separation across the blind spot is not sewn-up or the separation is sewn-up, but alignment thresholds are still high because of greater positional uncertainty around the blind spot.

If the blind spot is sewn-up in the cortex, the extent of contour interaction (in terms of degrees of visual angle) should be greater around the blind spot as compared to interaction across intact retina (assuming that the horizontal connections around the striate cortex's representation of the blind spot are similar in extents to the horizontal connections in other regions of the striate cortex). However, a significantly larger extent of interaction was not observed around the blind spot in any of the subjects. The issue is complicated by the fact that there is an amblyopic annulus about one deg wide around the blind spot (Le Grand, 1967) and there are thick blood vessels around the blind spot. These factors may have prevented the flanks from being as effective as they would be over intact retina. At flank separations of greater than $7 \mathrm{deg}$, these factors would have less of an influence, since the flanks would be well outside the amblyopic region and the concentration of blood vessels would be reduced. At these separations too, the interaction around the blind spot is not greater than interaction across intact retina. This argues against a "sewing-up" of the blind spot, at least as far as can be determined from contour interaction experiments.

\section{(D) Contour interaction in pathological scotomas}

Our results suggest that contour interaction can spread across the blind spot, in spite of the discontinuities in the retina and in the LGN's representation of the blind spot. We would expect to find similar contour interaction for retinal lesions if the sizes and eccentricities of the lesions are comparable to those of the blind spot. With cortical scotomas the contour interactions observed could depend on (among other things) the location of the cortical lesion involved, the stage of development at the inception of the scotoma and the time that has passed since the inception of the scotoma. Thus, contour interaction may be a useful tool to probe the nature and extent of dysfunction in pathological scotomas.

\section{REFERENCES}

Andrews, P. R. \& Campbell, F. W. (1991). Images at the blind spot. Nature, London, 353, 308
Andriessen, J. J. \& Bouma, H. (1976). Eccentric vision: Adverse interactions between line segments. Vision Research, 16, 71-78.

Beck, J. \& Halloran, T. (1985). Effects of spatial separation and retinal eccentricity on two-dot Vernier acuity. Vision Research, 25, $1105-1111$.

Bishop, P. O., Kozak, W., Levick, W. R. \& Vakkur, G. J. (1962). The determination of the projection of the visual field on to the lateral geniculate nucleus in the cat. Journal of Physiology, 163, 503-539.

Bouma, H. (1970). Interaction effects in parafoveal letter recognition. Nature, London, 226, 177-178.

Callaway, E. M. \& Katz, L. C. (1990). Emergence and refinement of clustered horizontal connections in cat striate cortex. Journal of Neuroscience, 10,1134-1153.

Chino, Y. M., Kaas, J. H., Smith, E. L. III, Langston, A. L. \& Cheng, H. (1992). Rapid reorganization of cortical maps in adult cats following restricted deafferentation in retina. Vision Research, 32, 789-796.

Drasdo, N. (1991). Neural substrates and threshold gradients of peripheral vision. In Kulikowski, J. J., Murray I. J. \& Walsh, V. (Eds), Vision and visual dysfunction (Vol. 5, pp. 250-264). New York: Macmillan.

Dow, B. M., Snyder, R. G., Vautin, R. G. \& Bauer, R. (1981). Magnification factor and receptive field size in foveal striate cortex of the monkey. Experimental Brain Research, 44, 213-228.

Fendrich, R., Wessinger, M. C. \& Gazzaniga, M. S. (1992). Residual vision in a scotoma: Implications for blindsight. Science, New York, 258, 1489-1491.

Ferree, C. E. \& Rand, G. (1912). The spatial values of the visual field immediately surrounding the blind spot and the question of the associative filling in of the blind spot. American Journal of Physiology, 29, 398-412.

Flom, M. C., Heath, G. G. \& Takahashi, E. (1963). Contour interaction and visual resolution: Contralateral effects. Science, New York, 142, 979-989.

Flom, M. C., Weymouth, F. W. \& Kahneman, D. (1963). Visual resolution and contour interaction. Journal of the Optical Society of America, 53(9), 1026-1032.

Gilbert, C. D. (1992). Horizontal integration and cortical dynamics. Neuron, 9, 1-13.

Gilbert, C. D. \& Wiesel, T. N. (1979). Morphology and intracortical projections of functionally identified neurones in cat visual cortex. Nature, London, 280, 120-125.

Gilbert, C. D. \& Wiesel, T. N. (1983). Clustered intrinsic connections in cat visual cortex. Journal of Neuroscience, 3 , 1116-1133.

Gilbert, C. D. \& Wiesel, T. N. (1989). Columnar specificity of intrinsic horizontal and corticocortical connections in cat visual cortex. Journal of Neuroscience, 9, 2432-2442.

Gilbert, C. D. \& Wiesel, T. N. (1990). The influence of contextual stimuli on the orientation selectivity of cells in primary visual cortex of the cat. Vision Research, 30, 1689-1701.

Gilbert, C. D. \& Wiesel, T. N. (1992). Receptive field dynamics in adult primary visual cortex. Nature, London, 336, 150-152.

Gilbert, C. D., Hirsch, J. A. \& Wiesel, T. N. (1990). Lateral interactions in visual cortex. Cold Spring Harbor Symposium on Quantitative Biology, LV, 663-677.

Hirsch, J. A. \& Gilbert, C. D. (1991). Synaptic physiology of horizontal connections in the cat's visual cortex. Journal of Neuroscience, $11,1800-1809$.

Horton, J. C. (1984). Cytochrome oxidase patches: A new cytoarchitectionic feature of monkey visual cortex. Philosophical Transactions of the Royal Society, London, 304, 199-253.

Hubel, D. H. \& Freeman, D. C. (1977). Projection into the visual field of ocular dominance columns in macaque monkey. Brain Research, 122, 336-343.

Hubel, D. H. \& Wiesel, T. N. (1974). Uniformity of monkey striate cortex: A parallel relationship between field size, scatter, and magnification factor. Journal of Comparative Neurology, 158, 295-306. 
Kaas, J. H., Guillery, R. W. Allman, J. M. (1973). Discontinuities in the dorsal lateral geniculate nucleus corresponding to the optic dise: A comparative study. Journal of Comparative Neurology, 147, $163-180$.

Kennedy, C., Des Rosiers, M. H., Jehle, J. W., Reivich, M., Sharpe F. \& Sokoloff, L. (1975). Mapping of functional neural pathways by autoradiographic survey of local metabolic rate with deoxyglucose. Science, New York, 187, 850-853.

Kennedy, C., Des Rosiers, M. H., Sakurada, O., Shinohara, O., Reivich, M., Jehle, J. W, \& Sokoloff, L. (1976). Metabolic mapping of the primary visual system of the monkey by means of the autoradiographic [it C]deoxyglucose technique. Pracedings of the National Acadamy of Science U.S.A., 73, 4230-4234.

Kooi, F. L, Toet, A., Tripathy, S. P. \& Levi, D. M. (1994). The effect of similarity and attention on contout interaction in peripheral vision. Spatial Vision. In press.

Le Grand, Y. (1967). Entoptic Phenomena, In Form and space vision (pp. 147-157). Bloomington: Indiana University Press.

LeVay, S., Connolly, M., Houde, J. \& Van Essen, D. C. (1985). The complete pattern of ocular dominance stripes in the striate cortex and visual field of the macaque monkey, Journal of Neuroscience, $5(2), 486-501$.

Levi, D. M., Klein, S. A. \& Aitsebaomo, A. P. (1985). Vernier acuity, crowding and cortical magnification. Viston Research, 25, 963-977.

Malpeli, J. G. \& Baker, F, H. (1975). The representation of the visual field in the lateral geniculate nucleus of Macaca mulata. Journal of Comparative Neurology, 161, 569-594.

Marrocco, R. T. \& McClurkin, J. W. (1979). Binocular interaction in the lateral geniculate nucleus of the monkey. Brain Research, 168 , 633-637.

Martin, K. A. C. \& Whitteridge, D. (1984). Form, function and intracortical projections of spiny neurones in the striate visual cortex of the cat. Journal of Physiology, 353, 463-504.

Nazir, T. A. (1992). Effects of lateral masking and spatial precueing on gap-resolution in central and peripheral vision. Vision Research, $32,771-777$.

Pearlman, A. L. (1987). The central visual pathways. In Moses, R. A. \& Hart, W. M. (Eds), Adler's physiology of the eye (pp. 583-618). St. Louis: Mosby.

Poggio, G. F. \& Fischer, B. (1977). Binocular interaction and depth sensitivity in striate and prestriate cortex of behaving thesus monkey. Journal of Neurophysiology, 40, 1392-1407.

Polat, U. \& Sagi, D. (1993). Lateral interactions between spatial channels: Suppression and facilitation revealed by lateral masking experiments. Vision Research, 33, 993-999.

Ramachandran, V. S. (1992). Blind spots. Scientific American, $266,86-91$

Ramachandran, V. S. \& Gregory, R. L. (1991). Perceptual filling in of artificially induced scotomas in human vision. Nature, London, 350 , 699 - 702.

Ramachandran, V. S., Gregory, R. L. \& Aiken, W. (1993). Perceptual fading of visual texture Borders. Vision Research, 33, 717-721.

Rodieck, R. W. \& Dreher, B. (1979). Visual suppression from nondominant eye in the lateral geniculate nucleus: A comparison of cat and monkey. Experimental Brain Research, 35, 465-477.

Sanderson, K. J., Bishop, P. O. \& Darian-Smith, 1. (1971). The properties of the binocular receptive fields of lateral geniculate neurons. Experimental Brain Research, 13, 178-207.

Schmielau, F. \& Singer, W. (1977). The role of visual cortex for binocular interactions in the cat lateral geniculate nucleus. Brain Research, 120, 354-361.

Sears, C. R. \& Mikaelian, H. H. (1994). An alternative hypothesis for perceptual completion surrounding the optic disk. Visual Neuroscience. In press.
Singer, W. (1970). Inhibitory binocular interaction in the lateral geniculate body of the cat. Brain Research, 18,165-170.

Smith, E. L., Chino, Y., Ridder, W. H., Kitagawa, K. \& Langston, A. (1990). Orientation bias of neurons in the lateral geniculate nucleus of macaque monkeys. Visual Neuroscience, 5, 525 -545 .

Sullivan, G. D., Oatley, K. \& Sutherland, N. S. (1972). Vernier acuity as effected by target length and separation. Perception and Psycho. physics, 12, 438-444.

Toet, A. \& Levi, D. (1992). The two-dimensional shape of spatial interaction zones in the parafovea. Vision Research, 32, 1349-1357.

Tolhurst, D. J. \& Ling, L. (1988). Magnification factors and the organization of the human striate cortex. Htman Nexrobiology, 6 , $247-254$.

Tripathy, S. P. \& Levi, D. M. (1993). Perceptual distortions and cortical binocular interactions around the blind spot. Imvestigative Ophthalmology \& Visual Science (Suppl.), 34/4, 794.

Tripathy, S. P., Levi, D. M. \& Ogmen, H. (1994). Length and separation distortions around the physiological blind spot. Vision Science and its Applications. Technical Digest SaES. In press.

Van Essen, D. C., Newsome, W. T. \& Maunsell, J. H. R. (1984). The visual field representation in striate cortex of the macaque monkey: Asymmetries, anisotropies, and individual variability. Vision Re. search, 24, 429-448.

Van Essen, D. C, DeYoe, E. A., Olavarria, J. F., Knierim, J. J., Fox, J. M., Sagi, D. \& Julesz, B. (1988). Neural responses to static and moving texture patterns in visual cortex of the macaque monkey. In Lam, D. M. K. \& Gilbert, C. D. (Eds), Proceedings of the retina research foundation symposia (Vol. 2, pp. 137-154). The Woodlands, Texas: Portfolio.

Westheimer, G. \& Hauske, G. (1975). Temporal and spatial interference with vernier acuity. Vision Research, 15,1137-1142.

Westheimer, G., Shimamura, K. \& McKee, S. (1976). Interference with line orientation sensitivity, Journal of the Optical Society of America, $66,332-338$.

Wiesel, T. N. \& Gilbert, C. D. (1988). Neural mechanisms of visual perception. In Lam, D. M. K. \& Gilbert, C. D. (Eds), Proceedings of the retina research foundation symposia (Vol. 2, pp. 7-33). The Woodlands, Texas: Portiolio.

Xue, J. T., Ramoa, A. S., Carney, T. \& Freeman, R. D. (1987). Binocular interaction in the dorsal lateral geniculate nucleus of the cat. Experimental Brain Research, 68, 305-310.

Acknowledgements-Supported by NEI grant R0IEY01728, NATO grant CRG890970. NEI grant P30EY07551, NAS Grant-in-aid of research through Sigma $\mathrm{Xi}_{\mathrm{i}}$. We thank the following for their helpful comments and suggestions and for critically reading earlier versions of the paper: Drs Harold Bedell, Yuzo Chino, Laura Frishman, Ron Harwerth, Frank Kooi, Haluk Ogmen, John Robson, Earl Smith III and Lex Toet. The technical assistance provided by Mr Chris Kuether and Ms Enita Torres is gratefully acknowledged. The patience of Ms Anh-Hong Doan who served as observer is greatly appreciated.

Note added in proof-Following submission we became aware of the recent work by Fiorani and colleagues [Fiorani, M., Rosa, M. G. P., Gattass, R. \& Rocha-Miranda, C. E. (1992) Dynamic surrounds of receptive fields in primate striate cortex: A physiological basis for perceptual completion? Proceedings of the National Academy of Science, U.S.A. 89, 8547-8551] which provides physiological evidence for binocular interactions in $\mathrm{Vl}$ in the region corresponding to the blind spot. 